\title{
Black-ish (ABC: 2014- ): el sueño (afro)americano Black-ish (ABC: 2014- ): The (Black) American Dream
}

\author{
Mayte DonstruP*
}

\section{RESUMEN}

Las series de televisión se han convertido en un objeto de estudio recurrente en el ámbito académico. En este sentido, se ha constatado que las narrativas televisivas funcionan como fábulas sociales que contienen en su seno moralejas sobre el funcionamiento de la sociedad. Este estudio articula un análisis de la serie Black-ish en torno al discurso ideológico; es decir, qué somos, qué hacemos y con qué objetivo (Van Dijk, 2009). Desde una perspectiva cualitativa, se ha realizado un análisis textual (McKee, 2002) que ha puesto de manifiesto que en la serie prevalecen los valores neoliberales. Al respecto, en la discusión se han contrapuestos los resultados con otras series del género y se ha corroborado la prevalencia del conservadurismo de las comedias de situación. Palabras clave: sitcom, nacionalismo, neoliberalismo, ideología, series de televisión, comedia.

\section{ABSTRACT}

Television series have become a recurring object of study in academia. Scholars have shown that television narratives function as social fables and morality plays about the functioning of society. This study analyzes the series Black-ish from the point of view of its ideological discourse: that is what we are, what we do, and what our objective is when we do it (van Dijk, 2009). Qualitatively speaking, the author has performed a textual analysis (McKee, 2002), showing that neoliberal values predominate in the series. In this regard, in the discussion, she compares the results from other series of the genre and shows the prevailing conservatism in sit-coms.

Key words: sit-com, nationalism, neoliberalism, ideology, television series, comedy.

\footnotetext{
*Universidad de Sevilla, <mdonstrup@us.es>.
} 


\section{INTRODUCCIÓN}

El estudio académico de las series de televisión es una tendencia en auge. En este sentido, se ha verificado que las narrativas de ficción sirven como explicación del funcionamiento de la sociedad y que su estudio clarifica cómo nos vemos a nosotros mismos como ciudadanos. Tal como argumenta Buonanno (1999), las series son fabuladoras, hablan del espectador y sobre sus experiencias en la vida cotidiana; son familiarizadoras, pues construyen un sentido común de la sociedad y aportan valores comunes a sus miembros; por último, sirven como mantenimiento de la comunidad, ya que considera aceptar los elementos novedosos paulatinamente, pero siempre rechazando todo lo considerado radical.

Considerando los presupuestos enunciados por Buonanno (1999), el presente análisis ambiciona arrojar luz sobre una de las series del género de comedia de situación: Black-ish (ABC: 2014- ), una producción focalizada en las vivencias de una familia afroamericana acomodada. En primer lugar, en este trabajo se realizará un repaso por la literatura científica relacionada con las temáticas de las comedias de situación, lo que nos permitirá adentrarnos posteriormente en la ficción televisiva objeto de estudio y discutir los valores ideológicos predominantes en la sitcom analizada.

\section{Series de teleVISIÓN E IDEOLoGíA}

Los productos de la cultura de masas pueden acoger en su seno discursos que abarcan todo el espectro ideológico (Pineda et al., 2018); sin embargo, cabe destacar que la mayoría de los que pertenecen al mainstream son de tinte conservador (Chomsky y Herman, 2009). En este sentido, producir un producto audiovisual con amplia cobertura y un mínimo de calidad implica un gran coste difícil de asumir; por ello, en última instancia son las grandes cadenas y productoras quienes deciden qué ve o qué no ve la luz. Una cuestión que no compete sólo al género informativo, pues, atendiendo a la consideración de Van Dijk sobre ideología “Quiénes somos, qué hacemos y con qué objetivo" (2009: 276), la ficción puede servir como un ideal vehículo ideológico. En efecto, los discursos -entre los que se incluyen los de ficción- son elementos generadores de conciencia por los que fluyen conocimientos y símbolos colectivos que interactúan en la sociedad (Jager, 2003). Singularmente, como veremos a continuación, las comedias de situación televisivas se prestan a tratar con una mayor soltura los temas sociales candentes. 


\section{COMEDIAS DE SITUACIÓN}

Brett Mills (2005) define el género comedia de situación (sitcom) como la narrativa en la que un conjunto establecido de personajes participa en situaciones cómicas recurrentes. Además de los protagonistas, Sergio Días Branco (2013) señala la importancia de los escenarios donde transcurren las tramas, resultado de una hibridación entre espacios internos y externos que el espectador reconoce con facilidad: "sitcoms may be alternatively described as a form of situating comedy. That is, a way of grounding and inscribing the comic idiosyncrasies and rapport of a small group of characters in a defined and regular spatial and temporal context" (2013: 95). Debido a sus particularidades, la comedia de situación es uno de los géneros más populares entre los espectadores y las cadenas de televisión, ya que ven en sus características estilísticas y formales un producto relativamente poco costoso de producir y con buenas expectativas mercadotécnicas (Staiger, 2000).

A nivel de contenido, gracias a su código humorístico, las comedias de situación pueden tratar con mayor éxito temas socialmente sensibles sin herir las sensibilidades de la audiencia (Staiger, 2000). A modo de ejemplo, los personajes de Seinfeld (19891998) se burlan de las convenciones masculinas heteronormativas y exploran formas alternativas de masculinidad (Miller, 2005). Sin embargo, también nos encontramos con comedias de situación que recurren a los tropos normativos y presentan a personajes con la necesidad de reafirmar su masculinidad como el reparto masculino de Friends (1994-2004) (Feasey, 2008). De hecho, una de las características más definitorias de las comedias de situación es su carácter conservador (Bonaut Iriarte y Del Mar Grandío Pérez, 2009; Bevan, 2013; Pugh, 2017; Novoa, 2018).

Laura Westengard (2017) explica cómo en 1950 las comedias tradicionales estadounidenses y británicas se alinearon con la tónica política de la guerra fría y trasmitieron en sus narrativas tópicos sociales occidentales conservadores. La encarnación de la familia tradicional perfecta en Leave it to a Beaver (1957-1963) o The Brady Bunch (1969-1974), por poner un par de ejemplos, conservaban los roles arquetípicos y evitaban insertar en sus tramas cuestiones raciales o sobre los derechos civiles (2017: 77). Asimismo, los productores y las cadenas marcaban unas pautas muy estrictas respecto a los comentarios o vestuarios que estaban permitidos enseñar al público; como señala Westengard (2017), "the financial conservatism at the heart of the network hesitation to produce the show is reflected in the ultimate conservatism of the show itself" (2017: 75).

Las comedias de corte realista no son las únicas que contienen marcas tradicionales. En este sentido, Alex Bevan (2013) expone la sitcom protagonizada por una simpática y atractiva bruja en Bewitched (1964-1972), quien encarnaba los roles tradicionales 
del matrimonio: perseguía ser la perfecta esposa y entregada ama de casa. Asimismo, es de destacar que dicha serie ha sido objeto de reprogramaciones y readaptaciones sucesivas en el tiempo, a modo de ejemplo, la película protagonizada por Nicole Kidman Bewitched (2005). Respecto al último punto, Bevan (2013) señala que las reformulaciones de las series de situación no sólo obedecen a motivos económicos, sino que existe un auge conservador en la sociedad: "Boomer sitcom remakes are about the popular nostalgic construction of the idyllic sitcom family and the way this image haunts ideas of nation and family to the exclusion of minority and working class voices" (Bevan, 2013: 317).

Por su parte, las comedias de situación contemporáneas no se alejan mucho de sus predecesoras. Por ejemplo, a pesar del aire transgresor de Modern Family (20092020), es característico que los roles patriarcales se mantengan intactos en la serie (Novoa, 2018): "Sitcoms are often accused of an inherent conservatism in their plotlines and structure, and so Modern Family allows viewers to gauge the tension between a program progressive in its ambitions and the narrative structures that might bend it to an ultimately conservative arc" (Pugh, 2017: 1). En adición, la posición socioeconómica de las familias protagonistas de dicha serie elimina de las tramas las desigualdades o discriminación social: "Its three major threats to the traditional nuclear family -homosexuality, divorce, and gender role reversal- are easily absorbed by the show's upper-middle class suburban gloss as well by each family member's commitment to the concept of family and togetherness" (Henneberg, 2016: 30).

\section{Objetivos}

Una vez expuestas las principales características de la comedia de situación, se propone como principal objetivo de esta investigación conocer los temas sociales sobre los que gira la serie Black-ish (2014- ). A partir del mencionado objetivo general, se ambiciona detectar los valores ideológicos de la comedia de situación analizada (por medio de las metas de los personajes y su definición de éxito) e interpretar el tratamiento de los asuntos raciales en su narrativa.

\section{Método}

En este estudio de caso se ha realizado una aproximación cualitativa a los mensajes ideológicos de la serie Black-ish (2014- ). Específicamente, se ha diseñado un análisis textual (McKee, 2002). Para la disección del producto audiovisual se han utilizado 
las escenas como unidades significativas de análisis "por su facilidad de identificación, y por gozar de sentido pleno en sí mismas desde el punto de vista del guion por su unidad temporal y de acción" (Grandío, 2011: 56). La base del análisis del discurso propuesto se ha situado sobre un eje: detectar los ideologemas que emite la serie. Los valores ideológicos se han extraído de dos manuales: Political ideologies. An Introduction (Heywood, 2012) e Ideologías y movimientos políticos contemporáneos (Antón y Torrens, 2012) con el fin de ejemplificar los conceptos y enfoques teóricos que se han ilustrado con las escenas de la serie. En cuanto al intervalo de tiempo, la muestra se ha basado en tres temporadas de la serie, compuestas de veinticuatro capítulos cada una. En el estudio de los personajes se ha tenido en consideración literatura especializada (Casseti y Di Chio, 2010; Brisset, 2011; Gibbs, 2002).

\section{Muestra de análisis}

El trabajo de investigación ha recogido un total de setenta y dos episodios de Black-ish como universo total de estudio. Con el fin de ilustrar los resultados, se ofrecen a continuación datos de interés de la trama general de la serie y los personajes.

La serie ha sido creada por Kenya Barris para ABC y se estrenó el 24 de septiembre de $2014 .{ }^{1}$ El idioma original de la serie es inglés, pero se encuentra disponible en la plataforma en español e inglés (versión doblada y / o subtitulada). En síntesis, con un reparto coral de ocho personajes, Black-ish narra la vida familiar de Andre (Dre) Johnson, un exitoso publicista afroamericano que lleva una vida acomodada en los suburbios de Los Ángeles:

Pues sí, soy el típico negro de toda la vida, guapo a rabiar y carismático que te mueres. Esta mulata con discapacidad pigmentaria que babea es mi mujer, Rainbow, y a pesar de la pinta que tiene ahora es médico. Somos afortunados: tenemos un casoplón, cuatro hijos y a mi padre. Esta vida no tiene nada que ver con mi origen, pues les prometí a mis padres que estudiaría, me graduaría y saldría echando leches de allí [...] Me encanta mi vida (Black-ish, 2014- : temp. 1, cap. 1).

\footnotetext{
${ }^{1} \mathrm{Al}$ comienzo de la segunda temporada los derechos de emisión fueron adquiridos por Amazon prime video, conglomerado que emite la serie desde entonces en el mercado internacional (ImdB, s.f.).
} 


\section{BLACK-ISH: INDIVIDUALISMO, COMPETENCIA Y MERITOCRACIA}

James Truslow Adams acuñó en The Epic of America el sueño americano como "the dream of a land in which life should be better and richer and fuller for every man, with opportunity for each according to his ability or achievement" (2012: 404). Por su parte, Hochschild (1995) identificó cuatro principios del American dream: 1) todos, sin importar su origen o estatus, pueden alcanzar el sueño americano, 2) siendo una esperanza de éxito 3) posible a través de acciones que están bajo el control directo individual; 4) por lo tanto, debido a las asociaciones de éxito y virtud, el sueño americano se hace realidad.

En la serie Black-ish, Dre Johnson se presenta ante el espectador como un ciudadano afroamericano estadounidense quien, a pesar de provenir de un barrio humilde, ha conseguido adquirir en la adultez una casa en una exclusiva urbanización: "para ser un chaval de barrio creo que estoy viviendo el sueño americano" (Black-ish, 2014- : temp. 1, cap. 1). El pasado con carencias económicas ha dejado lugar a un presente hedonista que no escatima en el dinero: estrenar zapatillas de marca a diario (temp. 1, cap. 1, temp. 3, cap. 3), preocupación por conseguir un tratamiento VIP en Disneyland (temp. 1, cap. 3) o los lujosos tratamientos de belleza de los cónyuges (temp. 3, cap. 4, temp. 3, cap. 23), entre otros, evidencian que uno de los axiomas de Black-ish es la exhibición del alto nivel de vida: “En Occidente somos consumistas, queremos lo mejor, lo más fardón y lo más grande. A veces se pone de moda lo más pequeño, pero al final lo grande siempre vuelve: todos consumimos, y aun a riesgo de que nos estereotipen, a los afroamericanos nos quema el dinero en la mano"' (temp. 2, cap. 13).

Ligado al concepto de sueño americano se encuentra el individualismo. Cabe recordar que el individualismo se refería originalmente a la igualdad de derechos, la libertad y la dignidad (Lukes, 1973). Sin embargo, en Estados Unidos la palabra individualismo fue pronto suplantada por otros términos como autosuficiencia y autocultura. En su repaso historiográfico, Lukes (1973) detectó que los escritos estadounidenses destacaban la necesidad de que los individuos cultivaran sus cualidades y capacidades únicas al margen de la sociedad. En la versión americana del individualismo romántico está la concepción implícita de que, a través de la expresión de un yo único y auténtico, un individuo encontrará satisfacción y gratificación personal (Becker y Marecek, 2008): "Individual freedom of choice, boundless opportunity, personal fulfillment, and happiness within every individual's reach -these constituents of positive psychology closely mirror what is taken to be the American Dream-" (2008: 1778). De hecho, en 1830 Alexis de Tocqueville mostró su preocupación por el individualismo estadounidense, pues incitaba erróneamente a pensar que "their whole destiny is 
in their own hands" (Tocqueville, 1994: 99). La inquietud de Toqueville se consume en la serie objeto de estudio como veremos a continuación.

Los protagonistas de Black-ish materializan el individualismo estadounidense y lo ensalzan de diferentes formas. En primer lugar, el Estado (las instituciones sociales) no cumple ningún papel en la serie: todos los miembros de la familia son autosuficientes y, es más, cumplen sus objetivos por sus propios méritos: "todo lo que he tenido lo he conseguido trabajando" (temp. 7, cap. 1). A pesar de una procedencia humilde, el protagonista enfatiza que ha podido triunfar en la vida sin ayuda social o contactos profesionales familiares: "yo me tuve que esforzar el doble para obtener lo que los enchufados tenían regalado; pero al final ese esfuerzo se vio recompensado y siempre he valorado lo que tengo porque sé que me lo he ganado" (temp. 3, cap. 9). Es más, cuando el benjamín de la familia obtiene los resultados de un test profesional y conoce que posee aptitudes para desarrollar labores en un grupo sindicalista de obreros, la idea de un hijo con una profesión sindical horroriza a los progenitores (temp. 6, cap. 3).

Por su parte, los escenarios exteriores donde se sitúa esta comedia de situación son la escuela, la comunidad de vecinos y los trabajos de los adultos (un hospital y una agencia de publicidad). Las relaciones de los personajes corales con los secundarios se encuentran basados en la competitividad y la desconfianza. Los protagonistas conciben a la vecindad como una competición donde el ganador debe exhibir sus triunfos sobre los demás: “Fijaos en esos padres tristes, ¿os podéis creer que tengo la misma edad que ese que tiene el móvil colgado del cinturón? La diferencia entre él y yo es que yo molo cantidubi" (temp. 1, cap. 17). Ante la posesión de una mayor cantidad de bienes materiales, Dre actúa desproporcionadamente con el padre de un compañero de Junior (su hijo) rompiendo toda relación que desequilibre su posición de superioridad económica (temp. 2, cap. 22). Incluso con la familia consanguínea prima el interés individual; este es el caso de la discusión por la herencia familiar de Dre con su hermana, Rhonda Johnson, quien aparentemente vive una vida más humilde que su hermano, pues trabaja como conductora para Uber y vive en un pequeño apartamento en la ciudad. A pesar de que Dre obtiene más ganancias, la hermana descubre que él se ha quedado con un apartamento que les correspondería a los dos, por lo que tuvo que existir una discusión para que él cediera y aceptase dividir la herencia entre ambos. Además, el trato con la vecina más recurrente de la serie, Janine, evidencia su mala relación con los vecinos en general; por ejemplo, ante su enfado por ser los únicos que no han sido invitados a una fiesta, Janine les expone que los escuchó insultarla una noche al volver del trabajo (temp. 2, cap. 14). Respecto al espacio laboral, Rainbow no mantiene ninguna relación de amistad con alguno de sus compañeros en el hospital, donde solo aparece interactuando con dos personajes: compitiendo por un ascenso y criticando la falta de sensibilidad de un doctor. 
Por otro lado, las conversaciones en la agencia de publicidad de Dre son más recurrentes. Las relaciones entre los publicistas se asemejan a transacciones económicas: existe una separación entre empleados por rangos ocupacionales (temp. 1, cap. 1) y competencia entre las personas por alcanzar un nuevo éxito. En el último caso, las competiciones pueden alcanzar el nivel de desleales sin que ello conlleve consecuencias para el personaje actante. A modo de ilustración, Dre en un capítulo se enfadó porque su compañero captó a un nuevo cliente, por lo que mediante técnicas desleales consiguió reemplazarlo en el último momento como líder de la nueva cuenta (temp. 1, cap. 20). No obstante, la sociedad en Black-ish posee un pegamento que une a todos los individuos independientemente de su posición socioeconómica y sus preferencias políticas: la nación.

¿Tú sabes lo que hace América grande? La unidad, sabemos cuando es necesario unir fuerzas para hacer realidad los sueños. Esta nación es un gran popurrí de personas comprometidas a que todos tengamos una vida mejor. $Y$ aunque tengamos mil formas de pensar, todos sabemos que, si persiste esa unidad, nuestro país persistirá, pero sólo si cada uno aporta lo suyo: eso es lo que hace grande esta nación (temp. 3, cap. 4).

A diferencia de otras ideologías como el liberalismo o el socialismo que se preguntan la relación entre el individuo, la sociedad y el Estado, el nacionalismo responde a la cuestión de quiénes son los individuos de una sociedad "fijando una relación de identidad entre el individuo, la sociedad-nación y el Estado" (Caminal, 2008: 171). Grosso modo,

el nacionalismo es una ideología que legitima la existencia y la permanencia del estado como nación, y que fundamenta, al mismo tiempo, la creación de naciones que afirman su derecho a la autodeterminación; en ambos casos, el nacionalismo se vale de la historia, de la cultura y de la educación, como instrumentos de cohesión y de proyección de identidades nacionales colectivas; en ambos casos, el nacionalismo se inscribe en procesos histórico-políticos en los que asume, bajo distintas formas, la representación política de un pueblo designado por aquél (Caminal, 2008: 184).

El nacionalismo es una combinación de patriotismo, es decir, "un apego afectivo profundamente sentido a la nación" (Conover y Feldman 1987: 1) o el "grado de amor y orgullo por la propia nación" (Kosterman y Feshbach 1989: 271) con la identidad jurídica, una vinculación del ciudadano por ley a un estado-nación jurídico: “El derecho es lo que da y garantiza la ciudadanía" (Caminal, 2008: 178). El patriotismo y los derechos / deberes jurídicos forman el nacionalismo con la idea de que el primero 
(la cohesión identitaria y cultural) facilite la adhesión voluntaria al ordenamiento jurídico de la nación.

Respecto al patriotismo, cabe distinguir entre dos vertientes: el acrítico y el constructivo. Schatz, Staub y Lavine definen el patriotismo acrítico como una falta de voluntad tanto para criticar como para aceptar la crítica a la nación (Schatz y Staub, 1997: 231; Schatz et al., 1999). El patriotismo acrítico está vinculado al autoritarismo, que se caracteriza por la tendencia a deferir a las figuras de autoridad y apoyarlas incondicionalmente (Schatz et al., 1999). Al contrario, el patriotismo constructivo se define como un apego al país caracterizado por la lealtad crítica y el cuestionamiento impulsados por un deseo de cambio positivo (Schatz et al., 1999: 153). En el patriotismo constructivo se encuentran afirmaciones como "Me opongo a algunas políticas de los Estados Unidos porque me preocupo por mi país y quiero mejorarlo" (Schatz et al., 1999). Las conversaciones en el hogar y en la agencia de Dre Johnson avalan que las marcas ideológicas de la serie corresponden al patriotismo constructivo. A modo de ejemplo, se expone en el siguiente discurso una declaración que corresponde a un episodio en el que los personajes discuten el resultado de las elecciones presidenciales de 2016, en las cuales resultó victorioso el magnate multimillonario Donald Trump:

Yo amo a este país, aunque a veces no me corresponda. Durante toda mi vida, a mis padres o a mis abuelos, este sistema ha ido en nuestra contra, pero seguimos haciendo lo posible por cumplir las reglas, aunque sepamos que nunca actuarán a nuestro favor. Hemos vivido en barrios a los que ni os acercaríais [...] La verdad que si le preguntase a un negro no diría que ganase quien ganase, su vida iría mejor, pero aun así votan, porque es lo que hay que hacer. Quiero mucho a este país y no quiero que se os olvide (temp. 3, cap. 12).

El capítulo se centra en el estado anímico de los personajes en la agencia de publicidad quienes se encuentran desanimados por los resultados electorales. No obstante, una trabajadora confiesa que ella y su familia votaron por Donald Trump porque las medidas durante la presidencia de Barack Obama no les dejaron conformes y necesitaban ver un cambio en la nación. Ante los reproches de los compañeros, Lucie (la votante de Donald Trump) expone su argumentario:

Por torpes que sean sus comentarios, al menos él dice lo que piensa. A saber qué piensa en realidad Hillary. Hillary no es un angelito... No soy una loca de extrema derecha, yo voté a Obama, convencí a mis padres republicanos para que le votaran y tras ocho años mi padre sigue en paro... Hillary dice que todo está bien y que no hay nada que cambiar, eso es mentira (temp. 3, cap. 12). 
El episodio en cuestión narra la polarización política de la sociedad estadounidense existente en el momento de la emisión del capítulo (año 2016) y da voz a ambos lados, concluyendo que lo importante para el país es mantenerse unido y dialogar para conseguir un consenso: “trabajemos juntos para hacer de este país una unión [...] más de cincuenta millones de personas vieron algo y no son locos, racistas [...] hay que dejar de insultarnos y dialogar" (temp. 3, cap. 12).

De hecho, en la primera temporada de la serie también se hace un intento por limar diferencias entre el partido Republicano y el Demócrata. Dicho episodio se centra en el interés de Junior por militar, ante el asombro de sus padres, en el partido Republicano. Los padres intentan convencerle de que los derechos que defienden los afroamericanos no concuerdan con los valores republicanos, pero los padrinos en el partido de Junior y el argumentario de su hijo son difíciles de refutar. En este sentido, Junior expone que el partido defiende los mismos valores que sus padres (esfuerzo y responsabilidad individual) y que, aunque no sean defensores públicos de las armas, todos los miembros de la familia poseen una (de hecho, Dre se compra una pistola en la segunda temporada). Por su parte, los padrinos de Junior en el Partido Republicano rebaten la creencia sobre el racismo de la organización: aunque existan radicales en el partido, éste abarca muchas personalidades y, fiscalmente, beneficia a la economía del país. Por tanto, más que los colores del partido que gobierne Estados Unidos, lo relevante en Black-ish es la unión por el amor patriótico a la nación. En el aspecto legal, en varias secuencias se advierte de la necesidad e importancia de las leyes para el correcto funcionamiento del país; por ejemplo, la participación de Dre como jurado (temp. 3, cap. 16) o las argumentaciones de Junior sobre los beneficios del orden y la disciplina: "Las reglas aportan certidumbre" (temp. 1, cap. 21).

Por último, uno de los axiomas sobre los que se asienta Black-ish es la tradición de las convenciones morales: el papel del hombre y la mujer en la estructura familiar son roles fijados que facilitan las rutinas. En este sentido, aunque ambos progenitores trabajen fuera del hogar, es Dre quien se ocupa de los temas económicos de la familia y es Rainbow quien se encarga del cuidado de los niños y del hogar (comidas, limpieza y todo lo asociado con rutinas privadas). Aunque el reparto de las tareas es un hecho que se detecta en todas las temporadas, la discusión de éstas surge en específico en el décimo tercer episodio de la segunda temporada. En dicho capítulo, los progenitores intentan un cambio de roles, pero Rainbow admite que siente pavor ante temas económicos y Dre no sabe lidiar con los asuntos del hogar, así que ambos retoman sus tradicionales funciones. 


\section{Black-ish: la tibieza sobre los temas raciales}

El racismo estructural de la sociedad estadounidense es un asunto que impregna toda la narrativa de Black-ish: la violencia policial, la falta de oportunidades y las trabas que se han impuesto históricamente a la comunidad afroamericana son expuestas narrativamente a través de argumentaciones y discusiones entre los personajes. Sin embargo, más que un problema con vigencia actual, la serie tiende a centrarse en los logros alcanzados contemporáneamente y alabar los derechos conseguidos por la comunidad. En este sentido, aunque Dre siempre exponga que llegar hasta donde ha llegado laboralmente le ha supuesto mucho esfuerzo, la cuestión es que lo ha conseguido, y que tiene a tres compañeros afroamericanos que también han alcanzado una posición relevante. Además, las relaciones entre personajes, independientemente de su color, se encuentran definidas por una posición socioeconómica elevada, y cuando se relacionan con personajes de color de posiciones marginales se cumplen los estereotipos de delincuencia y truhanería. Con un tono humorístico, los amigos de la infancia de Dre son representados como personajes picarescos que, de cierta forma, se aprovechan de la generosidad de su amigo. Por ejemplo, los padres, durante su infancia en un barrio marginal, robaban o mantenían acaloradas discusiones.

En adición, el miedo a la policía por parte de Dre se presenta como algo irracional y exagerado porque, de hecho, siempre que han aparecido las fuerzas del orden en la serie han actuado amablemente con el personaje. En particular durante una discusión en la familia sobre el exceso de veredictos de culpabilidad de las personas afroamericanas, Rainbow lanza una afirmación a favor de la justicia con datos estadísticos que muestran que hay más aciertos que fallos en el sistema judicial estadounidense. Cabe apuntar la amplia evidencia científica que señala el racismo institucional en el país, en el cual los afroamericanos son castigados sistemáticamente de una forma mucho más agresiva que los caucásicos (Quigley, 2012; Bell, 2017; Ezorsky, 2018).

En la serie, aunque no se reniega de algunos casos de violencia policial, Rainbow expone que el 75 por ciento de las personas negras contra las que se ha cometido violencia iban armadas y que la policía tiene derecho a defenderse. Como moraleja del episodio, Junior media una posición de reflexión que no condene a la estructura del país, "los policías desempeñan un papel importante en nuestra sociedad, pero con las miles de denuncias algo tiene que andar mal" (temp. 2, cap. 16). En la sociedad de Black-ish el racismo se encuentra tan superado que incluso tienen que inventarse situaciones para validar la denominada "culpabilidad blanca", un concepto que designa situaciones en las que se aprovechan de una supuesta posición de inferioridad.

En la tercera temporada, Zoe debe pasar las pruebas de la universidad, pero ha obtenido una baja calificación; ante ello, decide recurrir a la "culpabilidad blanca" y 
presenta ante la comisión de la Universidad una carta exponiendo el contexto de pobreza estructural donde vive inventándose una vida precaria por la que consigue una plaza. De este modo, y en contraposición con otras series como Orange is the New Black (2013-2019) (Iborra, 2018), el racismo en Black-ish es un problema superado en la actualidad.

\section{DISCUSIÓN DE LOS RESULTADOS Y CONCLUSIONES}

En los ochenta, el sueño afroamericano se materializaba en comedias de situación como The Cosby Show (1984-1992), donde una familia acomodada afroamericana perpetuaba los valores burgueses normativos (Frazer y Frazer, 1993; Westengard, 2017; Jhally y Lewis, 2019). De hecho, la familia de la serie (los Huxtable) aparece intertextualmente en Black-ish siendo categorizada como el modelo de familia afroamericana que anhelaba Dre Johnson (temp. 2, cap. 21). Al igual que en la serie objeto de este estudio, The Cosby Show referenciaba con tibieza los problemas raciales a los que se enfrenta la comunidad afroamericana y emulaba que el sueño americano está al alcance de la gran mayoría (Jhally y Lewis, 2019). Los estudios académicos sobre otra de las series referencia de los noventa como The Fresh Prince of Bel-Air (1990-1996) se encuentran divididos. Coleman (1998) y Gray (1995) denuncian la representación edulcorada de una comunidad afroamericana bien posicionada económicamente, mientras que Zook (1999) y Nyberg (2013) exponen que la representación de familias que se salen de la media social es un hecho positivo que posibilita la confrontación con los privilegios. No obstante, el individualismo predominante en las comedias de situación protagonizadas por afroamericanos mina los propósitos de justicia social: “Television's idealization of racial harmony, affluence and individual mobility is simply not within the reach of millions of Black Americans" (Gray, 1986: 239). De hecho, más allá de las observaciones realizadas por académicos, varios estudios de recepción han determinado que las audiencias afroamericanas se decantan por ficciones más plurales y realistas con su entorno (Clarence, 1999; Havens, 2001; Chitiga, 2003).

Así, aunque no todos los productos culturales sean ajenos a la crítica social (Romero, 2016), Black-ish se encuentra en concordancia con los temas ligeros y conservadores de las comedias de situación (Bonaut Iriarte y Del Mar Grandío, 2009; Bevan, 2013; Pugh, 2017; Novoa, 2018). En la serie analizada, el significado del éxito se encuentra anclado a las posesiones materiales y las metas de los personajes siempre van encaminadas a conseguir una mejor posición económica. Es más, cuando el protagonista ha percibido que podría perder una ganancia monetaria, no ha dudado en

apartar los valores comunitarios afroamericanos de los que presume y ha ejercido 
una competencia desleal contra sus compañeros (temp. 1, cap. 20). En Black-ish, el éxito se alcanza gracias al esfuerzo individual y las gratificaciones se disfrutan al margen de la sociedad, que solo es un escenario secundario portador de desconfianza. Según lo expuesto, en la serie nos encontramos con los cuatro axiomas individualistas y libertarios del sueño americano enunciados por Hochschild (1995): 1) proveniente de una familia humilde, Dre ha conseguido materializar el sueño americano 2) sin perder nunca la esperanza sobre sus posibilidades 3) mediante su propio esfuerzo y 4) virtud innata.

En definitiva, Dre Jonhson cumple el mito del hombre neoliberal: el sujeto hecho a sí mismo que ha triunfado gracias a su propio e individual esfuerzo. Son los valores de la competencia, la meritocracia y el individualismo los que enlazan Black-ish con el neoliberalismo. Las marcas ideológicas del neoliberalismo son el individualismo, la propiedad privada y la familia (Harvey, 2007: 29); la sociedad es una agrupación de sujetos que se mantienen unidos gracias a las transacciones materiales favorables. Existe en la hemeroteca una cita de la que fue primera ministra de Gran Bretaña, Margaret Thatcher, que resume la esencia del espíritu neoliberal: "you know, there's no such thing as society. There are individual men and women and there are families. And no government can do anything except through people, and people must look after themselves first" (The Guardian, 2013: 96). La familia y los individuos son los actantes de la narración en Black-ish, quienes actúan con intereses egoístas en aras de conseguir mayores beneficios económicos y desconfían de la sociedad que les rodea. No obstante, cabe añadir un pegamento que une a los sujetos que conviven en un mismo territorio: el nacionalismo. En este sentido, el amor a la nación surge en la serie como garante de las normas y las leyes sociales.

\section{FuENTES}

Adams, James Truslow

2012 The Epic of America, Boston, Routledge.

Antón, Joan y Xavier Torrens

2012 Ideologías y movimientos políticos contemporáneos, Madrid, Tecnos.

Becker, Danna y Jeanne MareceK

2008 "Dreaming the American Dream: Individualism and Positive Psychology. Social and Personality", Psychology Compass, no. 2, pp. 1767-1780, DoI: 10.1111 / j.1751-9004.2008.00139.x. 
Bell, Marcus

2017 "Criminalization of Blackness: Systemic Racism and the Reproduction of Racial Inequality in the U.S. Criminal Justice System", en Ruth Thompson Miller y Kimberley Ducey, eds., Systemic Racism: Making Liberty, Justice, and Democracy Real, Londres, Springer, 163-183.

Bevan, Alex

2013 "Archiving the Digital Transition in the Boomer TV Sitcom Remake", Adaptation, vol. 6, no. 3, pp. 305-319, en <https:/ / doi.org/10.1093/adaptation/apt007>.

Bonaut Iriarte, Joseba y María del Mar Grandío Pérez

2009 "Los nuevos horizontes de la comedia televisiva en el siglo xxI", Revista Latina de Comunicación Social, vol. 64, no.3, pp. 753-765, DOI: 10.4185/RLCS-642009-859-753-765.

BRISSET, D.

2011 Análisis filmico y audiovisual, Barcelona, voc.

Caminal, $\mathrm{M}$.

2008 “El nacionalismo”, en M. Caminal y X. Torrens, eds., Manual de ciencia política, Madrid, Tecnos, pp. 171-198.

Casetti, Francesco y Federico Di Chio

2010 Cómo analizar un film, Barcelona, Paidós.

Chitiga, Miriam

2013 "Black Sitcoms: a Black Perspective", Cercles, vol. 8, no. 1, pp. 45-68.

CHOMSKY, N. y E.S. HeRMAN

2009 Los guardianes de la libertad: propaganda, desinformación y consenso en los medios de comunicación de masas, Barcelona, Crítica.

Clarence, Lusane

1999 "Assessing the Disconnect between Television Audiences: The Race, Class, and Gender Politics of Married... With Children", Journal of Popular Film and Television, vol. 27, no. 1, pp. 12-20, DOI: 10.1080/01956059909602793. 
COLEMAN, RoBin

1998 African American Viewers and the Black Situation Comedy: Situating Racial Humor, Nueva York, Garland.

Conover, Pamela J. y Stanley Feldman

1987 “Memo to NEs Board of Overseers Regarding 'Measuring Patriotism and Nationalism'”, Italian Political Science Review, vol. 50, no. 1, en <http:/ / www. icpsr.umich.edu>.

Días Branco, SERGIO

2013 "Situating Comedy: Inhabitation and Duration in Classical American Sitcoms", en Steven Peacock y Jason Jacobs, eds., Television Aesthetics and Style, Londres, Bloomsbury, 93-103.

DIJK, T. A. VAN

2009 Discurso y poder. Contribuciones a los estudios críticos del discurso, Barcelona, Gedisa.

EZORSKY, GERTRUDE

2018 Racism and Justice: The Case for Affirmative Action, Nueva York, Cornell University Press.

FEASEY, ReBeCCA

2008 Masculinity and Popular Television, Edimburgo, Edinburgh University Press Books.

Frazer, June M. y Timothy C. Frazer

1993 "Father Knows Best and The Cosby Show: Nostalgia and the Sitcom Tradition", The Journal of Popular Culture, vol. 27, no. 1, pp. 163-172, DOI:10.1111/ j.0022-3840.1993.00163.x

Grandio, M. M.

2011 "Riesgo y trauma en la ficción televisiva estadounidense post 11-s: el caso de Heroes", en ZER-Revista de Estudios de Comunicación, vol. 16, no. 31, pp. 51-67.

Gray, HERMAN

1995 Watching Race: Television and the Struggle for Blackness, Londres, University Minneapolis Press. 
1986 "Television and the New Black Man: Black Male Images in Prime-time Situation Comedy", Media, Culture E Society, vol. 8, no. 2, pp. 223-242, en <https: / / doi.org/10.1177/016344386008002007>.

GuARDian, THE

2013 "Margaret Thatcher: A Life in Quotes", 8 de abril de 2013, en <https: / www. theguardian.com/politics / 2013/apr/08/margaret-thatcher-quotes>, consultada el 4 de marzo de 2021.

Harvey, D.

2007 Breve historia del neoliberalismo, Madrid, Tecnos.

\section{Havens, Timothy}

2001 "Subtitling Rap: Appropriating The Fresh Prince of Bel-Air for Youthful Identity Formation in Kuwait", International Communication Gazette, vol. 63, no. 1, pp. 57-72, en <https: / / doi.org/10.1177 / $0016549201063001005>$.

HenNeberg, SYLVIA

2016 "Rewriting the How-To of Parenting: What Is Really Modern about ABC'S Modern Family", Journal of Interdisciplinary Feminist Thought, vol. 9, no. 1, pp. 1-38.

HeYwOOd, ANDREW

2012 Political Ideologies. An Introduction, Londres, Red Globe Press.

HOCHSCHILD, JENNIFER

1995 Facing up to the American Dream: Race, Class, and the Soul of the Nation, Princeton, Princeton University Press.

Iborra Mallent, Juan Vicente

2018 "Black Lives Matter a través de las series de televisión estadounidenses", Norteamérica. Revista Académica del CISAN, UNAM, vol. 13, no. 2, pp. 279-297, en <https: / / doi.org/10.22201/ cisan.24487228e.2018.2.346>.

JAGER, S.

2003 “Discurso y conocimientos: aspectos teóricos y metodológicos de la crítica del discurso y el análisis de dispositivos", en R. Wodak y M. Meyer, comps., Métodos de análisis crítico del discurso, Barcelona, Gedisa, pp. 61-100. 
JHALly, Sut y Justin Lewis

2019 The Cosby Show, Audiences, and the Myth of the American Dream, Londres, Routledge.

Kosterman, Rick y Seymour Feshbach

1989 "Toward a Measure of Patriotic and Nationalistic Attitudes", Political Psychology, vol. 10, no. 2, pp. 257-74.

LUKES, STEVEN

1973 Individualism, Nueva York, Harper \& Row.

McKeE, A.

2002 Textual Analysis. A Beginner's Guide, Londres, SAGE.

Miller, Margo

2005 "Masculinity and Male Intimacy in Nineties Sitcoms: Seinfeld and the Ironic Dismissal", en James R. Keller y Leslie Stratyner, eds., The New Queer Aesthetic on Television: Essays on Recent Programming, Londres, McFarland, 147-159.

MiLLS, BRETT

2005 Television Sitcom, Londres, Birtish Film Institute.

Novoa Jaso, María Fernanda

2018 "La feminidad en la sitcom doméstica: representación y estereotipos. El caso de Modern Family", Revista Dígitos, vol. 4, no. 1, pp. 67-93.

NYBERG, FERDinAND

2013 "What Are You?: Negotiating Racial Authenticity and Imagining the Black Experience Through the Prism of Passing in The Fresh Prince of Bel-Air", ¡Plop! Revista de Pop, vol. 1, no. 2, pp. 45-67.

Pineda, A., J. D. Fernández-Gómez y A. Huici

2018 "Introducción: hacia un estudio sistemático de las ideologías políticas en la cultura de masas", en A. Pineda, J. D. Fernández-Gómez y A. Huici, coords., Ideologías políticas en la cultura de masas, Madrid, Tecnos, pp. 11-20. 
Pugh, Tison

2017 "Conservative Narrativity, Queer Politics, and the Humor of Gay Stereotypes in Modern Family", Camera Obscura, vol. 32, no. 3, pp. 1-31, en <https: / / doi.org/10.1215/02705346>.

Quigley, WiLliam

2012 "Racism: The Crime in Criminal Justice", Loyola Journal of Public Interest Law, vol. 13, no. 2, pp. 417-426.

Romero López, Alicia

2016 "Raza y género: violencia en The Bluest Eye (1970) de Toni Morrison”, Oceánide, no. 8, en <http: / / oceanide.netne.net/articulos/art8-1.pdf>.

SCHATz, Robert T. y ERvin STAUb

1997 "Manifestations of Blind and Constructive Patriotism: Personality Correlates and Individual-Group Relations", en Daniel Bar-Tal y Ervin Staub, eds., Patriotism: In the Lives of Individuals and Nations, Chicago, Nelson-Hall, 229-45.

Schatz, Robert T., Ervin Staub y Howard Lavine

1999 "On the Varieties of National Attachment: Blind versus Constructive Patriotism", Political Psychology, vol. 20, no. 1, pp. 151-174.

STAIGER, JANET

2000 Blockbuster TV: Must-See Sitcoms in the Network Era, Nueva York, New York University Press.

ToCQueVILle, Alexis DE

1994 Democracy in America, vol. II [1935-1840], Nueva York, Alfred A. Knopf.

WestengaRd, LAURA

2017 “The Brady Bunch: A Thoroughly Modern Family?”, en Aaron Barlow y Laura Westengard, eds., The 25 Sitcoms that Changed Television: Turning Points in American Culture, Westport, Praeger, pp. 71-81.

ZOOK, KRISTAL

1999 Color by Fox: the Fox Network and the Revolution in Black Television, Nueva York, Oxford University Press. 


\section{FUENTES VIDEOGRÁFICAS}

\section{Películas}

Bewitched. Dirigida por Nora Ephron. Estados Unidos: Columbia Pictures, Red Wagon Entertainment, 2005.

\section{SERIES DE TV}

Bewitched. Creada por Sol Saks. Estados Unidos: Ashmont Productions, Screen Gems Television, 1964-1972.

Black-ish. Creada por Kenya Barris. Estados Unidos: Wilmore Films / Artists First / Cinema Gypsy Productions / ABC Signature, 2014- .

Friends. Creada por David Crane y Marta Kauffman. Estados Unidos: Bright, Kauffman, Crane Productions / Warner Bros. Television, 1994-2004.

Leave it to a Beaver. Creada por Joe Connelly, Bob Mosher, Dick Conway. Estados Unidos: Gomalco Productions, Kayro-Vue Productions, Revue Studios, 1957-1963.

Modern Family. Creada por Steven Levitan y Christopher Lloyd. Estados Unidos: Levitan / Lloyd, 20th Century Fox Television, Picador Productions, 2009-2020.

Orange is the New Black. Creada por Jenji Kohan. Estados Unidos: Tilted Productions / Lionsgate Television, 2013-2019.

Seinfeld. Creada por Larry David y Jerry Seinfeld. Estados Unidos: West-Shapiro / Castle Rock Entertainment, 1989-1998.

The Brady Bunch. Creada por Sherwood Schwartz. Estados Unidos: Paramount Television, Redwood Productions, American Broadcasting Company (ABC), 19691974.

The Cosby Show. Michael Leeson. Estados Unidos: Bill Cosby Company / CarseyWerner Company, 1984-1992.

The Fresh Prince of Bel-Air. Creada por Andy Borowitz y Susan Borowitz. Estados Unidos: NBC Productions / Quincy Jones Entertainment / Quincy Jones-David Salzman Entertainment / The Stuffed Dog Company, 1990-1996. 